\title{
Calabi-Yau 簇的模问题与共形场论
}

\author{
谭福成 \\ (清华大学数学科学系, 北京 100084)
}

\begin{abstract}
摘要 从共形场论的观点来研究 Calabi-Yau 簇的模性质研究了一维 Calabi-Yau 轨形的情 形, 最终将它们所对应的模形式表达成 $\eta$ 函数的乘积通过对这些表达式的考察, 可以推测一 些高维 Calabi-Yau 簇的 $L$ - 级数. 希望这些结果对研究 Calabi-Yau 簇的模问题有所启发.
\end{abstract}

\section{关键词 $L$ - 级数 Calabi-Yau 簇 Gepner 模型}

$\operatorname{MSC}(2000)$ 主题分类 $53 \mathrm{C} 80$

\section{1 引言}

一直以来, 对 Calabi-Yau 流形的研究大多集中在几何、拓扑和弦理论等方面. 由于 Calabi-Yau 流形可以看作椭圆曲线的自然推广, 最近人们开始了对其算术性质的研究. 而 这方面的研究则主要集中于对有理数域上 Calabi-Yau 簇模猜想的证明.

Shioda 和 Inose $\mathrm{e}^{[1]}$ 研究了奇异 $K 3$ 曲面, 他们证明, 这些曲面可以定义在任意数域上. 之 后, Livné ${ }^{[2]}$ 证明了, 当这类曲面定义在有理数域上时, 相应的二维 Galois 表示对应于权为 3 的模形式. 在三维的情形下, 人们很自然的去考虑刚性 Calabi-Yau 簇, 因为这些簇的中间上 同调群是二维的. 这样一来, 由 Fontaine-Mazur 猜想 ${ }^{[3]}$, (可参看文献 [4]), 一个定义在有理数 域上的刚性 Calabi-Yau 三维簇的中间上同调的 $L$ - 级数与某个权为 4 的 cuspidal 模形式所 对应的 $L$ - 级数相同. Dieulefat 和 Manoharmayum ${ }^{[5]}$ 证明了, 在对那些坏约化的位上加上某 些限制的情形下, 有理数域上的刚性 Calabi-Yau 三维簇的模猜想成立. 当然, 也存在具有模 性质的非刚性 Calabi-Yau 三维簇, 比如说, 在文献 [6,7] 中就可以找到这样的例子. 关于这 方面最新进展的综述, 可参看文献 [8]. 在 $\mathrm{Cynk}$ 和 $\mathrm{Hulek}^{[9]}$ 的文章中, 他们构造了几个高维 Calabi-Yau 流形, 同时证明了这些流形的模猜想.

弦理论在物理上可以看作一个 Riemann 曲面上的二维共形场论. 数学上, 它可以来自对 应于一个仿射 Kac-Moody 代数的顶点算子代数, 因此, 弦紧化的问题可以解释为寻找 KacMoody 顶点代数与 Calabi-Yau 簇之间的对应. 文献 $[10,11]$ 用共形场论的观点, 具体地说, 用 弦理论的 theta 函数来解释 Fermat 曲线 $C_{3}$ 和 $C_{4}$ 的模问题.

本文处理一维 Calabi-Yau 轨形的模问题. 对于这些有理数域上的椭圆曲线, 得到了它们 所对应的模形式的 $\eta$ 函数的展开式, 结果如下所示:

$$
f\left(C_{3}, q\right)=\eta\left(q^{3}\right)^{2} \eta\left(q^{9}\right)^{2} ;
$$

收稿日期: 2006-05-17; 接受日期: 2007-08-13

E-mail: tanfch@gmail.com 


$$
\begin{aligned}
& f\left(E_{4}, q\right)=\eta\left(q^{4}\right)^{2} \eta\left(q^{8}\right)^{2} \otimes \chi_{2}(\cdot) ; \\
& f\left(E_{6}, q\right)=\eta\left(q^{6}\right)^{2} \eta\left(q^{6}\right)^{2} \otimes \chi_{3}(\cdot) .
\end{aligned}
$$

这一结果的细节会在第 4 节中详细阐述. 这些表达式显示, 应有某种规则把这些模形式与这 些 Calabi-Yau 轨形的 Gepner 模型联系在一起. 有一个观察可以用来作为证据支持这一法则, 那就是 Schoen 的五次簇. 我们已经知道, 这正是一个具有模性质的刚性 Calabi-Yau 三维簇. 应该还有更多这样的例子, 这一点会在后面加以解释.

可惜的是, 到目前为止所知的具有模性质的 Calabi-Yau 三维簇都不能表示成 Gepner 模 型, 这使得无法从共形场论的角度来解释这些有理数域上代数簇的模问题. 特别地, 用来描述 conifold 的形变, 比如 Schoen 五次簇, 我们既无法得到其 Gepner 模型, 也无法得到 KazamaSuzuki 模型. 不过, 采用非对角仿射不变量, 即 $D$ 型和 $E$ 型不变量, 的确可以找到能表示成 Gepner 模型的非 Brieskorn-Pham 型代数簇. 文献 [12] 对所有这些模型进行了分类, 而且存 在用 Kazama-Suzuki 构造所作的共形场论的推广. 文献 [13] 包含了相应的 Landau-Ginzburg 理论的具体构造.

\section{Calabi-Yau 簇的模性质}

\section{1 预备知识}

记 $K=\mathbb{F}_{q}$ 为一个有限域, 其中 $\operatorname{char} K=p$. 令 $X$ 是域 $K$ 上一个 $d$ 维几何连通的光 滑射影簇. 令 $\bar{K}$ 为 $K$ 的代数闭包, 记 $K$ 在 $\bar{K}$ 中唯一的 $n$ 次扩张为 $K_{n}$, 其中 $n \geqslant 1$. 记号 $c_{n}=\sharp X\left(K_{n}\right)$ 表示 $X$ 的 $K_{n^{-}}$有理点的数目. $X$ 的 zeta 函数定义如下:

$$
Z_{X}(t)=\exp \left(\sum_{n=1}^{\infty} \frac{c_{n}}{n} t^{n}\right) \in \mathbb{Z}[[t]] .
$$

正是为证明 Weil 猜想, Grothendieck, Artin 等提出了étale 理论. Deligne 由此最终完全 证明了这一猜想. 当然, 对这些 zeta 函数有理性的 $p$-adic 证明是首先由 Dwork 得到的.

定理 1 (Weil 猜想) 1. (有理性) $Z_{X}(t) \in \mathbb{Q}(t)$.

2. (Riemann 假设) 存在 $P_{0}, P_{1}, \ldots, P_{2 d} \in \mathbb{Z}[t]\left(P_{i}(0)=1\right)$, 使得

$$
Z_{X}(t)=\frac{P_{1}(t) P_{3}(t) \cdots P_{2 d-1}(t)}{P_{0}(t) P_{2}(t) \cdots P_{2 d}(t)} .
$$

如果做嵌入 $\overline{\mathbb{Z}} \hookrightarrow \mathbb{C}$, 并且做如下分解:

$$
P_{i}(t)=\prod_{j=1}^{\beta_{i}}\left(1-\alpha_{i, j} t\right), \quad \alpha_{i, j} \in \mathbb{C},
$$

则 $\left|\alpha_{i, j}\right|=q^{\frac{i}{2}}$.

3. (函数方程) 存在以下的函数方程:

$$
Z_{X}(t)\left(\frac{1}{q^{d} t}\right)= \pm q^{d \beta} t^{2 \beta} Z_{X}(t)
$$

其中

$$
\beta=\frac{1}{2} \sum_{i=0}^{2 d}(-1)^{i} \beta_{i} .
$$

令 $G=\operatorname{Gal}(\bar{K} / K)$ 是 $K$ 的抽象 Galois 群, 并令 $l$ 为一个不等于 $p$ 的素数. étale 上同 调 $H_{\mathrm{et}}^{m}\left(\bar{X}, \mathbb{Q}_{l}\right)$ 是 $G$ 的 $l$-adic 表示, 其中 $\bar{X}$ 是 $X$ 到 $\bar{K}$ 的基扩张. 我们有关于 $H_{\mathrm{e} t}^{i}\left(\bar{X}, \mathbb{Q}_{l}\right)$ 的 
Poincaré 对偶, 即完全配对

$$
H_{\text {ét }}^{i}\left(\bar{X}, \mathbb{Q}_{l}\right) \times H_{\text {ét }}^{2 d-i}\left(\bar{X}, \mathbb{Q}_{l}\right) \rightarrow \mathbb{Q}_{l} .
$$

于是, Frobenius 态射 $\operatorname{Frob}_{p}$ 诱导了 $H_{\text {êt }}^{i}\left(\bar{X}, \mathbb{Q}_{l}\right)$ 上的自同态 $\operatorname{Frob}_{p}^{*}$, 其特征多项式写成

$$
P_{i}^{(p)}(t)=\operatorname{det}\left(1-\operatorname{Frob}_{p}^{*} t \mid H_{\text {ét }}^{i}\left(\bar{X}, \mathbb{Q}_{l}\right)\right),
$$

这里 $t$ 是一个未定元. 事实上, 有

$$
P_{i}^{(p)}(t)=P_{i}(t)
$$

这就给出了 $L-$ 级数的概念.

定义 $1 \mathbb{F}_{q}$ 上的簇 $X$ 的第 $i$ 个 $L$ - 级数定义为

其中 $s$ 是一个复变量.

$$
L^{(i)}\left(X / \mathbb{F}_{q}, s\right)=\frac{1}{P_{i}^{(p)}\left(q^{-s}\right)},
$$

\subsection{Calabi-Yau 簇}

令 $X$ 是域 $K$ 上一个 $d$ 维的光滑射影簇.

定义 2 称 $X$ 是一个 Calabi-Yau 簇, 如果对任意的 $i(0<i<d)$ 都有 $H^{i}\left(X, \mathcal{O}_{X}\right)=0$, 并且 $X$ 的典则丛是平凡的.

注 1 当 $K$ 不是复数域 $\mathbb{C}$, 比如是某个数域的时候, 以上用来定义 Calabi-Yau 簇的不 变量是对该簇的复化而言的.

由于一个 Calabi-Yau 簇的几何亏格 $p_{g}(X)=h^{d, 0}$ 等于 1 , 可以看出, 一维的 Calabi-Yau 簇就是椭圆曲线, 而二维的 Calabi-Yau 簇是 $K 3$ 曲面. 三维的类似物就是我们非常感兴趣的 所谓 Calabi-Yau 三维簇. 由于复共轭和 Serre 对偶, 故得到

$$
h^{p, q}=h^{q, p}, \quad h^{p, q}=h^{d-p, d-q},
$$

于是一个 Calabi-Yau 三维簇 $X$ 的第 3 个 Betti 数可写成

$$
b_{3}=2\left(1+h^{2,1}\right) \text {. }
$$

定义 3 一个 Calabi-Yau 三维流形称为是刚性的, 如果 $h^{2,1}=0$.

于是, 一个刚性的 Calabi-Yau 三维流形的第 3 个étale 上同调就给出了秩为 2 的动形, 而 后者是非常重要的研究对象.

令 $X$ 是一个定义在数域 $K$ 上的 Calabi-Yau 簇, $G=\operatorname{Gal}(\bar{K} / K)$ 是 $K$ 的 Galois 群. 对 于素数 $\mathfrak{p} \in \mathcal{O}_{K}$, 记 $X$ 上的 Frobenius 态射为 Frob $_{p}$. 令 $q$ 为 $\mathfrak{p}$ 的范数, $l$ 是一个满足 $(l, q)=1$ 的素数. 若 $\mathfrak{p}$ 是一个好素数, 则 $\operatorname{Frob}_{p}$ 作用在 $l$-adic étale 上同调 $H_{\mathrm{e} t}^{i}\left(\bar{X}_{p}, \mathbb{Q}_{l}\right)$ 上, 其中 $X_{p}$ 是 $X$ 模 $\mathfrak{p}$ 的约化. 由于 $H_{\mathrm{ett}}^{i}\left(\bar{X}, \mathbb{Q}_{l}\right)=H_{\mathrm{ett}}^{i}\left(\bar{X}_{p}, \mathbb{Q}_{l}\right)$ (Grothendieck 的特殊化定理), 我们可以考虑 特征多项式

$$
P_{i}^{\mathfrak{p}}(t)=\operatorname{det}\left(1-\operatorname{Frob}_{p}^{*} t \mid H_{\text {ét }}^{i}\left(\bar{X}, \mathbb{Q}_{l}\right)\right) .
$$

若 $\mathfrak{p}$ 是一个不整除 $l$ 的坏素数, 可定义

$$
P_{i}^{\mathfrak{p}}(t)=\operatorname{det}\left(1-\operatorname{Frob}_{p}^{*} t \mid H_{\text {ét }}^{i}\left(\bar{X}, \mathbb{Q}_{l}\right)^{I_{p}}\right),
$$

这里 $I_{p}$ 是 $G$ 在 $\mathfrak{p}$ 处的惯性子群.

定义 4 设 $X$ 是一个定义数域 $K$ 上的 $d$ 维 Calabi-Yau 簇. 对整数 $i(0 \leqslant i \leqslant 2 d)$, 我们 可以定义 $X$ 的第 $i$ 个上同调 $L$ - 级数为 Euler 积

$$
L_{i}(X, s)=L\left(H_{\text {ét }}^{i}\left(\bar{X}, \mathbb{Q}_{l}\right), s\right)=\prod_{p \in \operatorname{SpecO}_{K}} P_{i}^{\mathfrak{p}}\left(q^{-s}\right)^{-1} .
$$


而 $X$ 的整体 $L$ - 级数则定义为

$$
L(X, s)=\prod_{i=0}^{d} L_{i}(X, s)^{(-1)^{i}} .
$$

有时候, 把中间的 $L$ - 级数 $L\left(H_{\mathrm{ett}}^{d}\left(\bar{X}, \mathbb{Q}_{l}\right), s\right)$ 称作 $X$ 的 $L$ - 级数.

\section{3 有理数域上 Calabi-Yau 簇的模问题}

为严格的陈述 Calabi-Yau 三维簇的模猜想, 首先考虑椭圆曲线的模问题. 一个有理数域 $\mathbb{Q}$ 上椭圆曲线 $E$ 的 $L$ - 级数形如

$$
L(E, s)=\prod_{p \text { 素数 }} \frac{1}{1-a(p) p^{-s}+\varepsilon(p) p^{1-2 s}} .
$$

如果记 $\Delta$ 是 $E$ 的判别式, 则有

$$
a(p)= \begin{cases}p+1-\sharp E\left(\mathbb{F}_{p}\right), & p \nmid \Delta, \\ \pm 1, & \text { 若 } p \text { 处的奇点是结点, } \\ 0, & \text { 若 } p \text { 处的奇点是尖点. }\end{cases}
$$

而 $\varepsilon(p)$ 等于 0 , 如果 $p$ 整除 $\Delta ;$ 否则等于 1 .

以下是由 Wiles 等 ${ }^{[14-16]}$ 证明的 Shimura-Taniyama-Weil 猜想.

定理 2 设 $E$ 是 $\mathbb{Q}$ 上的椭圆曲线, 其导子为 $N$, 则存在模形式 $f \in S_{2}\left(\Gamma_{0}(N)\right)$, 使得

$$
L(E, s)=L(f, s) .
$$

详细地说, 若 $f(q)=\sum_{n=1}^{\infty} a_{n}(f) q^{n}$, 则对任意的素数 $p$, 都有

$$
a(p)=a_{p}(f) .
$$

称定义在 $\mathbb{Q}$ 上的 $d$ 维 Calabi-Yau 簇 $X$ 有模性质, 若存在一个模形式 (或者更一般的, 自 守形式), 其对应的 Galois 表示同构于 $l$-adic Galois 表示

$$
\rho_{X, d}^{l}: G \rightarrow \operatorname{Aut}\left(H_{\text {ét }}^{d}\left(\bar{X}, \mathbb{Q}_{l}\right)\right) .
$$

特别地, $X$ 的 $L$ - 级数等于该模形式 (或自守形式) 的 $L$ - 级数, 二者最多相差有限个 Euler 因 子.

猜想 1 有理数域上的刚性 Calabi-Yau 三维簇有模性质.

注 2 有理数域上的刚性 Calabi-Yau 三维簇的模猜想可以看作 Fontaine-Mazur 猜想 ${ }^{[3]}$ 的几何实现, 同时也是关于二维 residual Galois 表示的 Serre 猜想 ${ }^{[17]}$ 的一个特殊情形.

下面更详细的描述 $\mathbb{Q}$ 上刚性 Calabi-Yau 三维簇的模猜想. 令 $t_{i}(p)=\operatorname{trace}\left(\operatorname{Frob}_{p}^{*} \mid H_{\mathrm{ett}}^{i}(\bar{X}\right.$, $\left.\mathbb{Q}_{l}\right)$ ). 由 Lefschetz 不动点公式, 有

$$
\sharp\left(X\left(\mathbb{F}_{p}\right)\right)=\sum_{i=0}^{6}(-1)^{i} t_{i}(p) .
$$

再利用几个对偶定理和 Riemann 假设, 得到

$$
t_{1}(p)=t_{5}(p)=0, \quad t_{0}(p)=1, \quad t_{6}(p)=p^{3}, \quad t_{4}(p)=p t_{2}(p),
$$

则给出了

$$
t_{3}(p)=1+p^{3}+(1+p) t_{2}(p)-\sharp X\left(\mathbb{F}_{p}\right) .
$$

在此情形下, 特征多项式

$$
P_{3}^{p}(t)=1-t_{3}(p) t+p^{3} t^{2} \in \mathbb{Z}[t] .
$$


$X$ 的 $L-$ 级数由局部 $L-$ 级数表示为

$$
L(X, s)=(*) \prod_{p \text { 好 }} \frac{1}{1-t_{3}(p) p^{-s}+p^{3-2 s}},
$$

其中 $(*)$ 是对应于坏素数的因子.

猜想 2 对于一个 $\mathbb{Q}$ 上的刚性 Calabi-Yau 三维簇, 存在一个模形式 $f \in S_{4}\left(\Gamma_{0}(N)\right)(N$ 只整除坏素数), 使得二维 Galois 表示 $\rho_{X, 3}^{l}$ 和对应于 $f$ 的表示有相同的半单化, 因此,

$$
L(X, s)=L(f, s) .
$$

进一步的, 对所有不整除 $N$ 的素数 $p$,

$$
t_{3}(p)=a_{p}(f) .
$$

注 3 到目前为止, 大约 30 个有模性质的刚性 Calabi-Yau 三维簇被找到（欲知这些例 子, 可参看 Yui 文献 [4]), 但是其中有些是彼此双有理等价的. 根据 Batyrev 的一个定理, 在不 计有些个乘积因子的意义下, 定义在数域上的光滑的极小 Calabi-Yau 三维簇的 $L$ - 级数是双 有理不变量. 作为一个推论, 维数不超过三的 Calabi-Yau 轨形的 $L$ - 级数是双有理不变量.

Schoen ${ }^{[18]}$ 考虑了 $\mathbb{P}^{4}$ 中的 Fermat 超曲面 $X_{5}$, 其定义方程为

$$
X_{0}^{5}+X_{1}^{5}+X_{2}^{5}+X_{3}^{5}+X_{4}^{5}=0 .
$$

这是一个非刚性的 Calabi-Yau 三维簇, 其 Hodge 数是 $h^{2,1}=101$. 对定义方程做形变得到

$Y=\left\{\left(X_{0}: X_{1}: X_{2}: X_{3}: X_{4}\right) \in \mathbb{P}^{4} \mid X_{0}^{5}+X_{1}^{5}+X_{2}^{5}+X_{3}^{5}+X_{4}^{5}-5 X_{0} X_{1} X_{2} X_{3} X_{4}=0\right\}$, 这是一个有 125 个结点的奇异簇. 分解奇点就得到一个刚性的 Calabi-Yau 三维簇, 记其为 $X$. Schoen 证明了

定理 3 存在一个 cusp 模形式 $f \in S_{4}\left(\Gamma_{0}(25)\right)$, 使得 $L(X, s)=L(f, s)$. 具体地说,

$$
\begin{aligned}
f(q)= & 25 \eta\left(q^{5}\right)^{4} \eta\left(q^{25}\right)^{4}+25 \eta(q) \eta\left(q^{5}\right)^{4} \eta\left(q^{25}\right)^{3}+20 \eta(q)^{2} \eta\left(q^{5}\right)^{4} \eta\left(q^{25}\right)^{2} \\
& +5 \eta(q)^{3} \eta\left(q^{5}\right)^{4} \eta\left(q^{25}\right)+\eta(q)^{4} \eta\left(q^{5}\right)^{4} .
\end{aligned}
$$

在这里 $\eta(q)=q^{1 / 24} \prod_{i=1}^{\infty}\left(1-q^{n}\right)$ 是 Dedekind eta 函数, 它是一个权为 $1 / 2$ 的模形式.

Dieulefait 和 Manoharmayum ${ }^{[5]}$ 得到了如下结果:

定理 4 设 $X$ 是一个 $\mathbb{Q}$ 上的刚性 Calabi-Yau 三维簇, 如果它满足以下某一个条件: (1) $X$ 在 3 和 7 处为好约化; (2) $X$ 在 5 处为好约化并且存在某个素数 $p \equiv \pm 2(\bmod 5)$, 使得 $t_{3}(p)$ 不被 5 整除, 则 $X$ 有模性质.

这个定理可用于 Schoen 的五次簇, 以及其他已知有模性质的刚性 Calabi-Yau 三维簇, 仅 有一个例外.

\subsection{Calabi-Yau 轨形及其 L- 级数}

除注 4 以外, 这一节关于 Calabi-Yau 轨形的处理主要参考了文献 [19].

定理 $5^{[20]}$ 设 $Y$ 是一个维数不大于 3 , 至多有 Abelian 奇点的的代数簇, 并假设其典则 丛是平凡的, 则存在 $Y$ 的消解 $\tilde{Y}$, 并且后者的典则丛也是平凡的.

有了这个定理, 就可以构造更多的 Calabi-Yau 簇.

定义 5 令 $F$ 是数域 $K$ 上一个 Fermat 超曲面, 其定义方程为 $X_{0}^{m}+X_{1}^{m}+\cdots+X_{d+1}^{m}=0$. 令 $W=\left(w_{0}, w_{1}, \ldots, w_{d+1}\right) \in \mathbb{N}^{d+2}$, 使得对任意 $i=0,1, \ldots, d+1, \operatorname{gcd}\left(w_{0}, \ldots, \widehat{w_{i}}, \ldots, w_{d+1}\right)$ $=1$ 并且 $w_{i} \mid m$. 我们有有限群概形 $\mu_{w_{i}}=\operatorname{Spec}\left(K[T] /\left(T^{w_{i}}-1\right)\right)$ 和

$$
\mu_{W}=\mu_{w_{0}} \times \mu_{w_{1}} \cdots \times \mu_{w_{d+1}} .
$$


$\mu_{W}$ 在 $\overline{\mathbb{Q}}$ 上的作用为

$$
\left(X_{0}, X_{1}, \ldots, X_{d+1}\right) \mapsto\left(\zeta_{w_{0}}^{e_{0}} X_{0}, \ldots, \zeta_{w_{d+1}}^{e_{d+1}} X_{d+1}\right),
$$

其中 $e_{i} \in \mathbb{Z} / w_{i} \mathbb{Z}, \zeta_{w_{i}}$ 是 $w_{i}$ 次本原单位根. 商

$$
Y=F / \mu_{W}
$$

是一个有 Abelian 奇点的簇, 它的消解称为 Calabi-Yau 轨形.

所有能够给出奇异 Calabi-Yau 簇的 $\langle m, W\rangle$ 如下所示:

定理 6 (文献 $[21,22]$ 和文献 [23] 当 $d=2$ ) 我们有

1. 当 $d=1$, 有三种情形 $\langle 3,(1,1,1)\rangle,\langle 4,(1,1,2)\rangle,\langle 6,(1,2,3)\rangle$.

2. 当 $d=2$, 有 14 种情形, 它们是 $\langle 4,(1,1,1,1)\rangle,\langle 6,(1,1,2,4)\rangle$, 等.

3 . 当 $d=3$, 有 147 种情形, 它们是 $\langle 5,(1,1,1,1,1)\rangle,\langle 6,(1,1,1,1,2)\rangle$, 等.

特别地, 以上得到的 Calabi-Yau 轨形都不是刚性的.

Goto $^{[23]}$ 和 Roan ${ }^{[24,25]}$ 分别对 $d=2$ 和 $d=3$ 时的奇异 Calabi-Yau 轨形的消解有显式 的描述. 结果显示, 出现在三维 Calabi-Yau 轨形的奇异轨迹中的曲线都是 $\mathbb{Q}$ 上光滑的加权 Fermat 曲线, 而所有一维 Calabi-Yau 轨形都在其中.

注意到 Shimura-Taniyama-Weil 猜想的证明对任意普通射影空间中定义在 $\mathbb{Q}$ 上的椭圆 曲线都适用, 而事实上, 任意的加权射影空间都可以嵌入到某个普通射影空间中去. 于是得到

定理 7 一个加权的二维射影空间中的任意一条定义在 $\mathbb{Q}$ 上的椭圆曲线都有模性质.

作为一个推论, 分别对应于权 $\langle 3,(1,1,1)\rangle,\langle 4,(1,1,2)\rangle,\langle 6,(1,2,3)\rangle$ 的加权椭圆曲线 $C_{3}, E_{4}$, $E_{6}$ 都有模性质.

注 4 (1) 在处理 $E_{4}$ 时应加以注意, 因为这条曲线上没有有理点. 我们应考虑它的 Jacobi $\operatorname{Jac}\left(E_{4}\right)$, 这是一条定义在 $\mathbb{Q}$ 上的带有区分点的椭圆曲线, 因此可用 Taniyama-Shimura-Weil 猜想. 这样就可以讨论 $E_{4}$ 的模问题, 因为

$$
L\left(\operatorname{Jac}\left(E_{4}\right), s\right)=L\left(E_{4}, s\right) .
$$

(2) 可以证明, 曲线 $C_{4}$ 的 Jacobi 簇 $J\left(C_{4}\right)$ 同源于椭圆曲线 $E_{4}$ 的三次方. 于是有

$$
L\left(C_{4}, s\right)=L\left(E_{4}, s\right)^{3} .
$$

考虑 $d$ 维 $m$ 次的 Fermat 超曲面 $F$, 这里 $m \geqslant d+2$. 记 $m$ 次单位根所成的群为 $\mu_{m}$, 对 角含入 $\mu_{m} \rightarrow \mu_{m}^{d+1}$ 的像为 $\Delta$. 商群 $\mu_{m}^{d+1} / \Delta$ 可以看作 $F$ 的自同构群 $\operatorname{Aut}(F)$ 的子群, 它的特 征标群 $\Gamma$ 由形如 $\left(\gamma_{0}, \ldots, \gamma_{d+1}\right) \in(\mathbb{Z} / m \mathbb{Z})^{d+1}$ 的元素构成, 其中 $\sum_{i=0}^{d+1} \gamma_{i} \equiv 0(\bmod m)$.

定义 $\Gamma$ 的一个子集 $\Lambda$ 为

$$
\Lambda=\left\{\left(\gamma_{0}, \ldots, \gamma_{d+1}\right) \in \Gamma \mid m \nmid \gamma_{i}, \forall i=0, \ldots, d+1\right\} .
$$

对每个权 $W=\left(w_{0}, \ldots, w_{d+1}\right)$, 定义 $\Lambda$ 的子集 $\Lambda(W)$, 其元素为 $\left(\gamma_{0}, \ldots, \gamma_{d+1}\right)$, 满足 $\gamma_{i} \in$ $w_{i} \mathbb{Z} / m \mathbb{Z}$.

令 $\mathfrak{p}$ 是分圆域 $\mathbb{Q}\left(\zeta_{m}\right)$ 中与 $m$ 互素的素理想. 于是 $\mathfrak{p}$ 的范数 $q \equiv 1(\bmod m)$, 存在唯一的 特征 $\chi_{\mathfrak{p}}: \mathbb{F}_{q}^{\times} \rightarrow \mu_{m}$, 满足 $\chi_{\mathfrak{p}}(x) \equiv x^{(q-1) / m}(\bmod \mathfrak{p})$, 对所有互素于 $\mathfrak{p}$ 的 $x \in \mathbb{Z}[\zeta] ; \chi_{\mathfrak{p}}(x)=0$ 对 所有 $x \equiv 0(\bmod \mathfrak{p})$.

对 $\lambda=\left(\lambda_{0}, \ldots, \lambda_{d+1}\right) \in \Lambda, d$ 维 Jacobi 和定义为

$$
j(\lambda)=(-1)^{d} \sum \chi_{\mathfrak{p}}\left(x_{1}\right)^{\gamma_{1}} \cdots \chi_{\mathfrak{p}}\left(x_{d+1}\right)^{\gamma_{d+1}},
$$

这里对 $\left(x_{1}, \ldots, x_{d+1}\right)$ 求和, 要求 $x_{i}(\bmod \mathfrak{p})$ 使得 $x_{1}+\cdots+x_{d+1}=-1$. 
定理 8 $^{[26]}$ 设 $F$ 是定义在 $\mathbb{Q}$ 上的 $d$ 维 $m$ 次 Fermat 超曲面. 线性变换 Frob ${ }_{p}^{*}$ 在 $H_{\text {êt }}^{d}\left(\bar{F}, \mathbb{Q}_{l}\right)$ 上的特征值可用 Jacobi 和 $j(\lambda)$ 来表达, 并且

$$
L(F, s)=L_{d}(F, s)=L_{\mathrm{Hecke}, d}(s)=(*) \prod_{\mathfrak{p} \nmid \delta} \prod_{\lambda \in \Lambda}\left(1-j(\lambda) q^{-s}\right)^{-1}
$$

是带 $\mathbb{Q}\left(\zeta_{m}\right)$ 的 Jacobi 和 Grössencharaktereas 的 Hecke $L$ - 级数, 其中 $\delta$ 是 Jacobi 和的导子, (*) 对应于那些坏素数.

定义 6 与权 $W$ 相容的带 Jacobi 和 Grössencharaktereas 的 Hecke $L$ - 级数定义为

$$
L_{\text {Hecke }, d}^{W}(s)=(*) \prod_{\mathfrak{p} \nmid \delta} \prod_{\lambda \in \Lambda(W)}\left(1-j(\lambda) q^{-s}\right)^{-1} .
$$

定理 $\mathbf{9}^{[4]}$ 令 $X$ 是一个定义在有理数域上、来自对应于 $\langle m, W\rangle$ 的奇异 Calabi-Yau 轨 形 $Y$ 的三维 Calabi-Yau 轨形, 则

$$
L(X, s)=(*) L_{\mathrm{Hecke}, 3}^{W}(s) \times \prod_{C} L(C, s-1)^{m_{c}},
$$

这里 $C$ 遍历 $Y$ 的奇异轨迹 $\Sigma(Y)$ 中所有不同的光滑不可约曲线, 其中 $m_{C}$ 是 $C$ 的重数.

进一步地, $L$ - 级数 $L(C, s)=L\left(H_{\mathrm{ett}}^{1}\left(\bar{C}, \mathbb{Q}_{l}\right), s\right)$ 可由与 reduced 权 $Q^{\prime}$ 相容的、带分圆域 $\mathbb{Q}\left(\zeta_{m^{\prime}}\right)$ 的 Jacobi 和 Grössencharaktereas 的 Hecke $L$ - 级数来表达. 而且 $m^{\prime}$ 和 $Q^{\prime}$ 可用 Roan 的算法具体的写出来.

\section{3 共形场论}

由带有 $N=2$ 超对称的有理恰当可解场论描述的共形场论给出了最简单的恰当可解 Calabi-Yau 簇. 我们考虑的是那些 Calabi-Yau 簇, 它们引出由某些 Virasoro 代数的扩张描述 的恰当可解模型, 这些 Virasoro 代数是由共形级为 $k$ 的 $\mathfrak{s u}(2)$ 仿射理论的流来定义的.

令 $\mathfrak{H}$ 为上半平面, 考虑 $\mathfrak{H} \times \mathbb{C}^{2}$ 上的共形场论特征. 众所周知, 这些特征可表示为

$$
\chi^{l}(\tau, z, u)=\sum_{\substack{m=-k+1 \\ l=m \bmod 2}}^{k} c_{l, m}^{k}(\tau) \Theta_{m}(\tau, z, u),
$$

其中

$$
c_{l, m}^{k}(\tau)=\frac{1}{\eta^{3}(\tau)} \sum_{\substack{-|x|<y \leqslant|x| \\(x, y) \text { or }\left(\frac{1}{2}-x, \frac{1}{2}+y\right) \\ \in \mathbb{Z}^{2}+\left(\frac{l+1}{2(k+2)}, \frac{m}{2 k}\right)}} \operatorname{sign}(x) e^{2 \pi i \tau\left((k+2) x^{2}-k y^{2}\right)}
$$

是弦函数, 而

$$
\Theta_{m}(\tau, z, u)=e^{-2 \pi i m u} \sum_{l \in \mathbb{Z}+\frac{k}{2 m}} e^{2 \pi i m l^{2} \tau+2 \pi i l z}
$$

是经典的 theta 函数. 记

$$
\Theta_{l, m}^{k}(\tau)=\eta(\tau)^{3} c_{l, m}^{k}(\tau)
$$

\section{1 量子维数}

$S$ - 生成元 $\tau \mapsto-1 / \tau$ 引出了关系

$$
\chi^{l}\left(-\frac{1}{\tau}, \frac{z}{\tau}, u+\frac{z^{2}}{2 \tau}\right)=e^{\pi i k z^{2} / 2} \sum_{m=0}^{k} S_{l m} \chi^{m}(\tau, u),
$$


这里模 $S$-matrix

$$
S_{l m}=\sqrt{\frac{2}{k+2}} \sin \left(\frac{(l+1)(m+1) \pi}{k+2}\right) .
$$

定义 7 量子维数是定义为

$$
Q_{l}=\frac{S_{l 0}}{S_{00}}
$$

的那些实数. 广义上, 定义级为 $k$ 的 $\mathfrak{s u}(2)$ 理论的量子维数为

现在考虑手性基本场

$$
Q_{l m}=\frac{S_{l m}}{S_{0 m}} .
$$

$$
\phi_{l} \phi_{m}=\sum_{r} N_{l m}^{r} \phi_{r}
$$

的熔解法则. 广义的量子维数是熔解矩阵 $N_{l}$ 的特征值, 因而是某个代数数域 $K$ 中的代数整 数, 这是因为 $N_{l}$ 各位置上的元素均为整数. Boer 和 Goeree 证明了, 由仿射 $\mathfrak{s u}(2)_{k}$ 理论的量 子维数定义的域扩张 $K / \mathbb{Q}$ 是 Abelian 扩张. 于是由 Kronecker-Weber 定理可知, $K$ 包含在分 圆域 $\mathbb{Q}\left(\mu_{m}\right)$ 中, $m$ 为某个整数.

由于量子维数是实数, 故应考虑由 $\left(\xi_{m}+\xi_{m}^{-1}\right)$ 生成的实子域. 在 $m$ 次 Fermat 超曲面的 情形下, 量子维数取值在实域 $\mathbb{Q}(\sqrt{m}) \subset \mathbb{Q}\left(\mu_{m}\right)$ 中, 而后者恰好是由定理 8 给出的分圆域.

\subsection{Gepner 模型}

Gepner 曾建议, 由张量几个 $N=2$ 极小模型得到的恰当可解的弦紧化, 在经过适当的投 影后, 应该给出在一定程度上相关于 Fermat 型 Calabi-Yau 簇的几何紧化的理论.

例如, 我们可得到五次簇 $X_{5} \subset \mathbb{P}^{4}$ 的 Gepner 模型为

$$
X_{5} \sim\left(\mathrm{SU}(2)_{k=3, A_{1}}\right)_{\mathrm{GSO}}^{\otimes 5},
$$

其中 $A_{1}$ 表示 $\mathfrak{s u}(2)$ 分拆函数的对角不变量, 而 GSO 表示投射, 后者保证了态的整的 $U(1)-$ 电量.

\section{4 从共形场论的观点看模问题}

\subsection{Fermat 三次曲线}

由标准的算法 (这里使用了 William Stein 的数据库 ${ }^{[27]}$ ) 我们可以得到, 对应于椭圆曲线 $C_{3}$ 的模形式 $f\left(C_{3}, q\right) \in S_{2}\left(\Gamma_{0}(27)\right)$ 的 $q-$ 展开为

$$
f\left(C_{3}, q\right)=q-2 q^{4}-q^{7}+5 q^{13}+4 q^{16}-7 q^{19}+\cdots,
$$

这里 $q=e^{2 \pi i \tau}$.

以下结果给出了模形式 $f\left(C_{3}, q\right)$ 的一个弦理论意义上的解释, 所用的是由一个仿射 $\mathfrak{s u}(2)$ Kac-Moody 代数决定的恰当可解共形场论的特征.

定理 10 ${ }^{[10]}$ 模形式 $f\left(C_{3}, q\right)$ 分解为

$$
f\left(C_{3}, q\right)=\Theta_{1,1}^{1}\left(q^{3}\right) \Theta_{1,1}^{1}\left(q^{9}\right),
$$

这里 $\Theta_{1,1}^{1}(\tau)=\eta^{3}(\tau) c_{1,1}^{1}(\tau)$ 是对应于有理数域的二次扩张 $\mathbb{Q}(\sqrt{3})$ 的 Hecke 不定模形式, 它由 共形级为 $k=1$ 的仿射 $\mathfrak{s u}(2)$ 代数唯一的弦函数 $c_{1,1}^{1}(\tau)$ 决定.

证明 为证明此定理, 首先注意到一个事实, 即 $c_{1,1}^{1}(\tau)=\eta^{-1}(\tau)$ 推出 $\Theta_{1,1}^{1}(\tau)=\eta^{2}(\tau)$, 这 是一个权为 1 的模形式. 模形式 $f\left(C_{3}, q\right)$ 和 $\Theta_{1,1}^{1}\left(q^{3}\right) \Theta_{1,1}^{1}\left(q^{9}\right)$ 都属于 $S_{2}\left(\Gamma_{0}(27)\right)$, 这允许使用 
Faltings 和 Serre 的一般结果: 如果两个模形式截止到前面某一项都相同, 则这两个模形式相 同. 对那些关于同余子群 $\Gamma_{0}(N)$ 的模形式, 在文献 [28] 中得到这个数为 $k\left[\Gamma_{0}(1): \Gamma_{0}(N)\right] / 12$. 在当前的例子中, 容易算出这个数为 6 , 而这两个权为 2 的模形式的前六项的确相同. 这样就 证明了定理.

注 5 三次曲线 $C_{3}$ 出现在对应于 $\langle 12,(1,1,2,4,4)\rangle$ 的 Calabi-Yau 三维簇 $X_{12}$ 的奇异轨 迹中. 由于 $C_{3}$ 和 $X_{12}$ 的 Gepner 模型分别为

$$
\mathbb{P}_{2} \supset C_{3} \sim\left(\mathfrak{s u}(2)_{k=1, A_{1}}\right)_{\mathrm{GSO}}^{\otimes 3}
$$

和

$$
\mathbb{P}_{(1,1,2,4,4)} \supset X_{12} \sim\left(10_{A_{1}}^{2} \otimes 4_{A_{1}} \otimes 1_{A_{1}}^{2}\right)_{\mathrm{GSO}},
$$

定理 10 给出了级为 1 的两个极小因子的特征的几何构造.

最后, 通过计算曲线 $X_{0}(27)$ 的亏格, 知道空间 $S_{2}\left(\Gamma_{0}(27)\right)$ 是一维的, 因此 $\Theta_{1,1}^{1}\left(q^{3}\right) \Theta_{1,1}^{1}\left(q^{9}\right)$, 在不计常数因子的意义下, 是 $S_{2}\left(\Gamma_{0}(27)\right)$ 的唯一生成元.

\section{2 加权的四次椭圆曲线}

当前的情形和处理 $C_{3}$ 时的主要区别在于, 此时共形级为 2 , 于是有多个弦函数. 然而结 果表明, 跟我们的讨论相关的是 theta 函数 $\Theta_{1,1}^{2}(q)$, 它是一个权为 1 的模形式.

定理 $11^{[11]}$ 对应于椭圆曲线 $E_{4}$ 的模形式 $f\left(E_{4}, q\right)$ 分解为有扭的乘积

$$
f\left(E_{4}, q\right)=\Theta_{1,1}^{2}\left(q^{4}\right)^{2} \otimes \chi_{2}(\cdot),
$$

其中扭特征为 Legendre 符号 $\chi_{2}(\cdot)=\left(\frac{2}{2}\right)$, 而 $\Theta_{1,1}^{2}(\tau)$ 是弦函数 $c_{1,1}^{2}(\tau)$ 的仿射 $\mathfrak{s u}(2)$ theta 函 数.

注 6 严格说来, 上面的模形式 $f\left(E_{4}, s\right)$ 是对应于椭圆曲线

$$
\widetilde{E_{4}}: v^{2}=u^{3}+u
$$

的模形式, 而这条曲线同源于 $E_{4}$. 事实上, 变换

$$
\begin{aligned}
& v=X Z / Y, \\
& u=-(X / Y)^{2}
\end{aligned}
$$

就是一个从 $E_{4}$ 到 $\widetilde{E_{4}}$ 的同源. 这里, $E_{4}$ 的定义方程为 $X^{4}+Y^{4}+Z^{2}=0$.

定理 11 的证明类似于 $C_{3}$ 的情形, 因为 $f\left(E_{4}, q\right)$ 和 $\Theta_{1,1}^{2}(4 \tau)^{2} \otimes \chi_{2}$ 都是空间 $S_{2}\left(\Gamma_{0}(64)\right)$ 中的模形式. 需要注意的是, $\chi_{n}(\cdot)$ 的导子为 $n$, 若 $n \equiv 1(\bmod 4) ;$ 为 $4 n$ 若 $n \equiv 2,3(\bmod 4)$.

对平方自由的 $n$, Legendre 特征可用来刻画有理素数 $p$ 在二次扩张 $\mathbb{Q}(\sqrt{n}) / \mathbb{Q}$ 中的分歧 行为. 这一刻画就把特征 $\chi_{2}(\cdot)$ 和二次域 $\mathbb{Q}(\sqrt{2})$ 联系起来. 注意到级为 2 的 $\mathfrak{s u}(2)$ 理论的量 子维数也取值在 $\mathbb{Q}(\sqrt{2})$ 中. 在三次椭圆曲线 $C_{3}$ 的情形下, 量子维数取值在 $\mathbb{Q}$ 中, 这是有理 数域的平凡扩张.

注 7 (1) 物理上, 正如 Lynker ${ }^{[11]}$ 指出的, 特征 $\chi_{2}(\cdot)$ 是量子维数的生成域的弦理论解 释, 因此提供了弦理论谱的几何解释.

(2) 数学上, Legendre 符号的出现则来自于 $E_{4}$ 定义方程的第 3 个分量上的 $\mathbb{Z} / 2 \mathbb{Z}-$ 作用.

在当前的情形下, 可仿照从前的办法算出空间 $S_{2}\left(\Gamma_{0}(32)\right)$ 的维数等于 1 , 于是 $\Theta_{1,1}^{2}\left(q^{4}\right)^{2}$ 是它唯一的生成元, 最多相差一个常数因子. 


\section{3 加权的六次椭圆曲线}

回忆一下, 只存在 3 个一维的 Calabi-Yau 轨形, 列示如下:

$$
\begin{aligned}
& C_{3}=\left\{(X: Y: Z) \in \mathbb{P}^{2} \mid X^{3}+Y^{3}+Z^{3}=0\right\}, \\
& E_{4}=\left\{(X: Y: Z) \in \mathbb{P}_{(1,1,2)} \mid X^{4}+Y^{4}+Z^{2}=0\right\}, \\
& E_{6}=\left\{(X: Y: Z) \in \mathbb{P}_{(1,2,3)} \mid X^{6}+Y^{3}+Z^{2}=0\right\} .
\end{aligned}
$$

我们可以分别得到以下相应的 Gepner 模型:

$$
\begin{aligned}
C_{3} & \sim\left(\mathfrak{s u}(2)_{k=1, A_{1}}\right)_{\mathrm{GSO}}^{\otimes 3}, \\
E_{4} & \sim\left(\mathfrak{s u}(2)_{k=2, A_{1}}\right)_{\mathrm{GSO}}^{\otimes 2}, \\
E_{6} & \sim\left(\mathfrak{s u}(2)_{k=4, A_{1}} \otimes \mathfrak{s u}(2)_{k=1, A_{1}}\right)_{\mathrm{GSO}} .
\end{aligned}
$$

前两节已经从共形场论的角度讨论了前两条椭圆曲线的模问题. 下面, 处理加权椭圆曲 线 $E_{6}$ 模问题, 仍然结合共形场论来考虑.

取 $v=Z / X^{3}, u=Y / X^{2}$, 得到椭圆曲线

$$
v^{2}=-u^{3}-1,
$$

它同源于 $E_{6}$, 因而它的 $L$ - 级数与 $E_{6}$ 相同.

再次利用 William Stein 的数据库, 得到对应于椭圆曲线 $v^{2}=-u^{3}-1$ 的模形式 $f\left(E_{6}, q\right) \in$ $S_{2}\left(\Gamma_{0}(144)\right)$ 的 $q$ - 展开. 当然, 这也是椭圆曲线 $E_{6}$ 的模形式的 $q$ - 展开. 具体为

$$
f\left(E_{6}, q\right)=q+4 q^{7}+2 q^{13}-8 q^{19}-5 q^{25}+4 q^{31}-10 q^{37}-8 q^{43}+O\left(q^{48}\right) .
$$

由于最终要应用 Serre 和 Faltings 的定理, 故只列出展开式的前 48 项, 这样已经足够了.

为把模形式 $f\left(E_{6}, q\right)$ 用来自仿射 $\mathfrak{s u}(2)$ 理论的弦函数的 theta 函数来表示, 首先要知道 哪些 theta 函数应该出现在表达式中. 在当前的情形下, 有两个共形级, $k=3$ 和 $k=1$, 这使 得备选的 theta 函数太多, 难以选择.

但是, 注意到由 $\mathrm{Kac}$ 和 Perterson ${ }^{[29]}$ 的结果, theta 函数 $\Theta_{1,1}^{1}$ 和 $\Theta_{1,1}^{2}$ 可以用 eta 函数的 乘积表出如下:

$$
\begin{aligned}
& \Theta_{1,1}^{1}(q)=\eta(q)^{2}, \\
& \Theta_{1,1}^{2}(q)=\eta\left(q^{2}\right) \eta\left(q^{4}\right) .
\end{aligned}
$$

这样, 就可以把之前的模形式也用 eta 函数的乘积写下来.

$$
\begin{aligned}
& f\left(C_{3}, q\right)=\eta\left(q^{3}\right)^{2} \eta\left(q^{9}\right)^{2}, \\
& f\left(E_{4}, q\right)=\eta\left(q^{4}\right)^{2} \eta\left(q^{8}\right)^{2} \otimes \chi_{2}(\cdot) .
\end{aligned}
$$

将把这些表达式与相应的 Gepner 模型

$$
C_{3} \sim\left(\mathfrak{s u}(2)_{k=1, A_{1}}\right)_{\mathrm{GSO}}^{\otimes 3}
$$

和

$$
E_{4} \sim\left(\mathfrak{s u}(2)_{k=2, A_{1}}\right)_{\mathrm{GSO}}^{\otimes 2}
$$

做一下比较, 就可以看到内在的规则.

具体地说, 对以上任意一个模形式, 都取 $k+2$ 作为 $q$ 在第 1 个 eta 函数中的幕次, 其中 $k$ 是对应于该模形式的 Gepner 模型的最高共形级. 而第 2 个 $q$ 的次数是 $k+2$ 与该 Gepner 
模型中 $\mathfrak{s u}(2)$ 出现次数的乘积. 以此为依据, 我们猜测模形式 $f\left(E_{6}, q\right)$ 等于 $\eta\left(q^{6}\right)^{2} \eta\left(q^{6}\right)^{2}$, 最 多差一个 Dirichlet 特征作为扭部分.

事实上, 我们有

$$
\eta\left(q^{6}\right)^{2} \eta\left(q^{6}\right)^{2}=q-4 q^{7}+2 q^{13}+8 q^{19}-5 q^{25}-4 q^{31}-10 q^{37}+8 q^{43}+O\left(q^{48}\right) .
$$

把它和 $f\left(E_{6}, q\right)$ 的 $q$ - 展开相比较, 我们发现符号的变化发生在 $q^{7}, q^{19}, q^{31}$ 和 $q^{43}$ 这几项之前. 这暗示我们, Legendre 符号 $\chi_{3}(\cdot)$ 应出现在表达式中.

定理 12 对加权的椭圆曲线 $E_{6}$, 存在其模形式的 $\eta$ - 展开

$$
f\left(E_{6}, q\right)=\eta\left(q^{6}\right)^{2} \eta\left(q^{6}\right)^{2} \otimes \chi_{3}(\cdot),
$$

这里 $\chi_{3}(\cdot)$ 是对应于二次域 $\mathbb{Q}(\sqrt{3})$ 的 Legendre 符号.

证明 由之前的构造可以看到, 方程的两边都是 $S_{2}\left(\Gamma_{0}(144)\right)$ 中的模形式. 计算它们的 前 48 项, 再加以比较, 就会发现两边完全相同. 于是由 Serre 和 Faltings 的定理, 两个模形式 相同相等, 证毕.

注 8 (1) Legendre 符号 $\chi_{3}(\cdot)$ 的出现是因为 $\mathfrak{s u}(2)_{k=4}$ 的量子维数取值在二次域 $\mathbb{Q}(\sqrt{3})$ 中, 这一点已经从共形场论的角度给出了解释. 而在数学上, 该 Legendre 符号包含了在构造 Calabi-Yau 轨形时的群作用的信息.

(2) 由于 $\Theta_{1,1}^{1}(q)=\eta(q)^{2}$, 还可得到另一表达式

$$
\left.f\left(E_{6}\right), q\right)=\Theta_{1,1}^{1}\left(q^{6}\right) \Theta_{1,1}^{1}\left(q^{6}\right) \otimes \chi_{3}(\cdot) \in S_{2}\left(\Gamma_{0}(144)\right) .
$$

这个表达式会让人觉得是 Gepner 模型中的最低共形级 $k=1$ 决定了哪个弦理论的 theta 函 数应该作为相应模形式的组成部分, 而最高共形级仅仅决定了 Legendre 符号.

如前, 注意到 $\eta\left(q^{6}\right)^{2} \eta\left(q^{6}\right)^{2}$ 是空间 $S_{2}\left(\Gamma_{0}(36)\right)$ 的唯一生成元, 因为后者的维数为 1.

\section{4 总结}

通过以上的分析和讨论, 得到一维 Calabi-Yau 轨形的模性质的整体图像, 其观点则来自 于共形场论. 现列示如下:

$$
\begin{aligned}
& f\left(C_{3}, q\right)=\eta\left(q^{3}\right)^{2} \eta\left(q^{9}\right)^{2} ; \\
& f\left(E_{4}, q\right)=\eta\left(q^{4}\right)^{2} \eta\left(q^{8}\right)^{2} \otimes \chi_{2}(\cdot) ; \\
& f\left(E_{6}, q\right)=\eta\left(q^{6}\right)^{2} \eta\left(q^{6}\right)^{2} \otimes \chi_{3}(\cdot) .
\end{aligned}
$$

我们认为, 在 Calabi-Yau 簇和共形场论的对应当中, 起主要作用的是相应的仿射 $\mathfrak{s u}(2)$ 理 论的最高共形级. 第 2 节中介绍的 Schoen 的五次簇 $X$ 可以作为我们这一观察的佐证. 该代 数簇是来自于 Fermat 超曲面 $X_{5}$ 的刚性 Calabi-Yau 三次簇. 尽管目前还没有办法把 Schoen 五次簇 $X$ 的 Gepner 模型完全写下来, 但是我们却可以猜测模形式 $f(X, q)$ 的 $q$ - 展开的领头 项, 这是因为超曲面 $X_{5}$ 的 Gepner 模型为

$$
\left(\mathfrak{s u}(2)_{k=3, A_{1}}\right)_{\mathrm{GSO}}^{\otimes 5} .
$$

而领头项的系数 25 可能来自于形变项 $-5 X_{0} X_{1} X_{2} X_{3} X_{4}$ 以及奇异 Calabi-Yau 簇 $Y$ 的 125 个结点.

致谢 感谢周坚教授长期以来的指导和鼓励. 同时感谢 Victor Kac 教授和 Rolf Schimmrigk 教授在本文准备过程中解答了一些有益的问题. 


\section{参 考 文 献}

1 Shioda T, Inose H. On singular K3 surfaces. In: Complex Analysis and Algebraic Geometry. Cambridge: Cambridge University Press, 1977

2 Livné R. Motivic orthogonal two-dimensional representations of Gal( $(\overline{\mathbb{Q}} / \mathbb{Q})$. Israel J Math, 92(1-3): 149-156 (1995)

3 Fontaine J M, Mazur B. Geometric Galois Representations. In: Coates J, Yau S-T eds. Elliptic Curves, Modular Forms, and Fermat's Last Theorem. Hong Kong: International Press, 1995

4 Yui N. Update on the modularity of Calabi-Yau varieties. Fields Inst Commun, 38: 307-362 (2003)

5 Dieulefait L, Manoharmayum J. Modularity of rigid Calabi-Yau threefolds over $\mathbb{Q}$. Fields Inst Commun, 38: 159-166 (2003)

6 Hulek K, Verrill H. On modularity of rigid and nonrigid Calabi-Yau varieties associated to the root lattice A4. Nagoya Math J, 179: 103-146 (2005). arXiv: Math.AG/0304169

7 Hulek K, Verrill H. On the modularity of Calabi-Yau threefolds containing elliptic ruled surfaces. arXiv: math.AG/0502158

8 Meyer C. Modular Calabi-Yau Threefolds. In: Fields Institute Monograph Series. Providence, RI: American Mathematical Society, 2005

9 Cynk S, Hulek K. Construction and examples of higher-dimensional modular Calabi-Yau manifolds. arXiv: Math AG/0509424

10 Schimmrigk R, Underwood S. The Shimura-Taniyama Conjecture and conformal field theory. J Geom Phys, 48: 169-189 (2003)

11 Lynker M, Schimmrigk R. Geometric Kac-Moody Modularity. arXiv: hep-th/0410189

12 Lynker M, Schimmrigk R. A-D-E quantum Calabi-Yau manifolds. Nucl Phys B, 339: 121-157 (1990)

13 Lynker M, Schimmrigk R. String compactifications on G/H Landau-Ginzburg theories. Phys Lett B, 253: 83-89 (1991)

14 Wiles A. Modular elliptic curves and Fermat's last theorem. Ann Math, 141: 443-551 (1995)

15 Taylor T, Wiles A. Ring-theoretic properties of certain Hecke algebras. Ann Math, 141: 553-572 (1995)

16 Breuil C, Conrad B, Diamond F, et al. On the modularity of elliptic curves over $\mathbb{Q}$. J Amer Math Soc, 14: 843-939 (2001)

17 Serre J P. Sur les représentations modulaires de degré 2 de Gal $(\overline{\mathbb{Q}} / \mathbb{Q})$. Duke Math J, 54: 179-230 (1987)

18 Schoen C. On fiber products of rational elliptic surfaces with section. Math Z, 197: 177-199 (1988)

19 Yui N. The $L$-series of Calabi-Yau Orbifolds of CM type. Preprint, 1998

20 Greene B, Roan S S, Yau S T. Geometric singularities and spectra of Landau-Ginsburg models. Comm Math Phys, 142: 245-259 (1991)

21 Yui N. The $L$-series of certain Calabi-Yau orbifolds over number fields. Preprint, 1997

22 Yui N. Arithmetic of certain Calabi-Yau varieties over number fields. Arithmetic and Geometry of Algebraic Cycles. NATO Sci Ser, 548: 519-565 (2000)

23 Goto Y. Arithmetic of certain weighted disgonal surfaces over finite fields. J Number Theory, 59: 37-81 (1996)

24 Roan S S. On Calabi-Yau orbifolds in weighted projective spaces. Intern J Math, 1: 273-310 (1990)

25 Roan S S. The mirror of Calabi-Yau orbifolds. Intern J Math, 2: 439-455 (1991)

26 Weil A. Jacobi sums as "Grössencharaktere". Trans Amer Math Soc, 73: 487-795 (1952)

27 Stein W. http://modular.fas.harvard.edu/Tables/index.html

28 Sturm J. On the congruence of modular forms. In: Number Theory, Chudnovsky D V, Chudnovsky G V, Cohn H, et al., eds. LNM Vol. 1240. New York: Springer, 1987

29 Kac V, Peterson D. Infinite-dimensional Lie algebras, theta functions and modular forms. Adv Math, 53: 125-264 (1984) 